\title{
Innate immune memory: implications for development of pediatric immunomodulatory agents and adjuvanted vaccines
}

\author{
Ofer Levy ${ }^{1,2}$ and Mihai G. Netea ${ }^{3,4}$
}

Unique features of immunity early in life include a distinct immune system particularly reliant on innate immunity, with weakThelper (Th)1-polarizing immune responses, and impaired responses to certain vaccines leading to a heightened susceptibility to infection. To these important aspects, we now add an increasingly appreciated concept that the innate immune system displays epigenetic memory of an earlier infection or vaccination, a phenomenon that has been named "trained immunity." Exposure of neonatal leukocytes in vitro or neonatal animals or humans in vivo to specific innate immune stimuli results in an altered innate immune set point. Given the particular importance of innate immunity early in life, trained immunity to early life infection and/or immunization may play an important role in modulating both acute and chronic diseases.

\section{INTRODUCTION}

Immune responses consist of a complex system of cellular and humoral components that recognize self from nonself, eliminate the latter, thereby realizing a crucial function of neutralization of invading pathogenic micro-organisms. These defense mechanisms are essential for the survival and perpetuation of all multicellular organisms. The adaptation of immune responses to a previously encountered infection, in order to respond with an increased efficacy upon reinfection, gives a distinct evolutionary advantage to the host. In humans, this function is fulfilled by specific immune responses such as the production of antibodies (Abs) or the generation of T-cell clones specific towards a particular pathogen. These responses are also known as adaptive immunity, because they occur as an adaptation to infection by that pathogen. Adaptive immunity often confers lifelong protection to reinfection with the same pathogen through the rapid clonal expansion of memory $\mathrm{T}$ and/or B cells that induce a rapid and effective response (1).

Importantly, specific immune responses during a primary infection require several days and up to $2 \mathrm{wk}$ to develop an effective antimicrobial effect. The host defense during these first days of infection is assured by innate immunity. In contrast to $\mathrm{T}$ and B cells, the cells of the innate immune system such as granulocytes, macrophages, or natural killer $(\mathrm{NK})$ cells are immediately available to fight efficiently and kill a broad range of pathogens, but it has been thought that they do not confer specificity or immunological memory to the innate host defense.

Remarkably, the last few years of research have dramatically changed the dogma of innate immunity being "nonspecific." Indeed, the different classes of pattern recognition receptors (PRRs) such as the Toll-like receptors (TLRs), C-type lectin receptors, nucleotide-binding oligomerization domain-like receptors, and retinoic acid-inducible gene-I (RIG-I) helicases present on leukocytes surfaces are responsible for the semispecific activation of cells of the innate immune system through the specific recognition of pathogen-associated molecular patterns of pathogenic micro-organisms (2). Moreover, in addition to providing early defense against infections, the innate immune response plays an essential role in triggering and driving the acquired immune system to respond effectively to infection $(3,4)$. Moreover, the dogma of innate immunity lacking memory has also started to be questioned (5). Several studies during the last decades showed enhanced immune responses upon reinfection in invertebrates lacking adaptive immunity, such as cockroaches (6), shrimps (7), or mealworm beetles (8). Interestingly, invertebrates also demonstrated enhanced secondary responses (9) and transmission of protection to offspring (10). Recently, Witteveldt et al. showed that such innate immune memory might even be used for the vaccination of invertebrates (11).

The adaptive characteristics of innate immunity, enabling it to respond with enhanced efficiency during a reinfection, are evident in vertebrates as well. Enhanced heterologous (often also referred to as "nonspecific") innate immune protection can be mediated by epigenetic reprogramming of innate immune cells and has been called trained immunity (12). The discovery of trained immunity may change our understanding of the scope and nature of immunological responses.

\section{SUMMARY OF CURRENT EVIDENCE FOR THE TRAINED IMMUNITY PHENOMENON}

Evidence for the trained immunity has been found in plants, invertebrates, (e.g., insects) and, more recently, mammals (see Table 1). Somatic rearrangement of immunological receptors is used by vertebrates to induce adaptive immune responses (13), while alternative splicing of pattern-recognition genes is employed by the host defense of invertebrates to 


\section{Implications of innate immune memory $\mid$ ReV'ew}

Table 1. Studies demonstrating the presence of memory characteristics of innate immunity in plants, invertebrates, and mammals

\begin{tabular}{|c|c|c|c|c|}
\hline Organism & Infection type & Biological effect & Specificity & Mechanism \\
\hline \multicolumn{5}{|c|}{ Plants_-"systemic acquired resistance" } \\
\hline Plants (refs. 49-53) & Viruses, bacteria, fungi & Protection against reinfection & Variable & Salicylic acid \\
\hline \multicolumn{5}{|l|}{ Nonvertebrates-Insects } \\
\hline Beetle (ref. 8) & $\begin{array}{l}\text { LPS, or bacterial } \\
\text { prechallenge }\end{array}$ & Protection against reinfection & - & Transgenerational priming \\
\hline \multirow{2}{*}{ Drosophila (ref. 54) } & B. bassiana & & & \\
\hline & S. marcescens & & & \\
\hline Anopheles gambiae (ref. 55) & Midgut flora & Protection against Plasmodium & + & $\begin{array}{l}\text { Toll-dependent hematocyte-differentiation } \\
\text { factor }\end{array}$ \\
\hline \multicolumn{5}{|c|}{ Mammals_"trained immunity" } \\
\hline Mice (refs. 16,20) & Hypersensitization & & & Hepatic CXCR6 ${ }^{+}$NK cells \\
\hline Humans (ref. 27) & BCG vaccination & $\begin{array}{l}\text { Protection against nonrelated } \\
\text { infections }\end{array}$ & - & Monocyte reprogramming \\
\hline
\end{tabular}

confer adaptation to infection (14). For example, Anopheles gambiae mosquitoes use alternative splicing of the immunoglobulin (Ig)-domain coding gene "Down syndrome cell adhesion molecule" (Dscam) to produce a highly diverse set of $>31,000$ potential alternative splice forms, which enable specific recognition and protection against bacteria and parasites (15). Thus, both somatic rearrangement and alternative splicing result in a receptor repertoire that is sufficiently diverse to discriminate between broad varieties of different pathogens and provide adaptive functions to the immune system.

In addition to the mechanisms that lead to a broad panel of pathogen-recognition receptors, a second type of adaptive immune response has been recently described, in which functional reprogramming of innate immune cells induces an increased response to a secondary infection. The function of the prototypic innate immune NK cells can be enhanced by a primary viral infection, leading to protection against reinfection with viral pathogens (16-18). Infection of mice with cytomegalovirus induced proliferation of NK cells bearing the virus-specific Ly49H receptor. After a contraction phase at the end of the infection, these NK cells reside in lymphoid organs for several months and can rapidly degranulate and produce cytokines upon viral reinfection (16). In addition, after hapten sensitization, long-lived NK cells are responsible for the contact hypersensitivity response, independent of B and T lymphocytes (19). Moreover, memory NK cells can also develop adaptive immunity to structurally diverse antigens, an activity that requires the chemokine receptor CXCR6 (20). Overall, memory is increasingly considered an important characteristic of NK-cell responses.

In addition to NK cells, monocytes/macrophages also display adaptive characteristics. Multiple murine studies have demonstrated that immunization with the anti-tuberculosis vaccine Bacille Calmette-Guérin (BCG) protects not only against mycobacteria, but also against infections with Listeria monocytogenes, Salmonella typhinirium, Staphylococcus aureus (S. aureus), Candida albicans (C. albicans), or Schistosoma mansoni (21-24). Interestingly, T-cell-independent mechanisms mediated by activated tissue macrophages contribute to this protection $(21,23,24)$. In addition, in children in West Africa, BCG vaccination has been shown to confer heterologous beneficial effects with decreased morbidity due to infections other than tuberculosis, and decreased overall mortality $(25,26)$. As the protection against other infections reported in these studies is heterologous ("nonspecific"), it is unlikely that a specific adaptive immune response is the protective mechanism responsible. Indeed, it has been proposed that epigenetic reprogramming of monocytes, based of modulation of histone methylation profiles, leads to an enhanced function of monocytes and heterologous ("nonspecific") protection to infections induced by BCG vaccination (27).

In addition to BCG vaccination, other specific challenges with live, attenuated agents have produced heterologous responses. After injection of an attenuated nongerminative strain of C. albicans, mice were protected not only against a virulent C. albicans strain but also against the Gram-positive bacterium S. aureus (28). Such protection is also induced in athymic mice, indicative of a T-cell-independent mechanism (29). Protection against reinfection is dependent on typical innate immune host response mechanisms such as macrophages (28) and the production of proinflammatory cytokines (30). The molecular mechanism of this protection is mediated by the dectin-1/Raf-1 pathway activated by $\beta$ glucans on the cells wall of Candida, leading to a "primed" epigenetic profile based on specific hypermethylation of histones (31). Thus, epigenetic reprogramming of monocytes is a key novel feature of innate immune memory, (trained immunity), and is likely to be of general importance after certain infections or immunizations. 


\section{EVIDENCE THAT TRAINED IMMUNITY OCCURS IN EARLY LIFE}

Several in vitro studies suggest that the innate immune system has memory features in early life, indeed right from birth. Stimulation of human newborn cord blood mononuclear cells in vitro, for example with Staphylococcus epidermidis, enhances expression of a variety of PRRs including TLRs, providing a potential mechanistic basis for greater innate immune responses to subsequent challenges (32).

Both murine and human in vivo studies have indicated that stimulation of the neonatal innate immune system can alter the set point of innate immunity such that responses to subsequent stimuli are altered. Intraperitoneal administration of TLR agonists (TLRAs) to newborn mice enhance subsequent cytokine- and phagocyte-based responses to bacterial infection with associated improvement in survival from polymicrobial sepsis induced by intraperitoneal injection of a cecal slurry (33). Of note, bloodstream infection in critically ill preterm human newborns is associated with enhancement of pathogen-specific mononuclear cell PRR expression in the setting of Gram-positive bacteremia (TLR2, MyD88) and Gram-negative bacteremia (TLR4, MD-2, and MyD88) (34). A novel newborn murine model of intravenous infection with S. epidermidis in the first $24 \mathrm{~h}$ of life confirms selective TLR2 upregulation in liver mRNA (35). These observations suggest that the neonatal innate immune system can remember previous activation such that responses to subsequent microbial challenge are altered.

Further evidence that early life trained immunity could be clinically relevant in vivo is provided by a recent study indicating that histologic chorioamnionitis, known to be associated with increased TLR expression in the chorioamniotic membranes (36), is associated with a reduced risk of late onset bacterial sepsis, including a lower risk of S. epidermidis bacteremia (37). Along these lines, among the smallest, most premature infants, early onset sepsis (i.e., that within the first $72 \mathrm{~h}$ of life), is associated with a diminished risk of late onset sepsis (i.e., that occurring after $72 \mathrm{~h}$ of life) (38). As early onset sepsis and late onset sepsis can be caused by distinct bacteria, this clinical observation raises the possibility that innate immune engagement during early onset sepsis enhances innate immune responses such that the risk of late onset sepsis is diminished.

Immunization of human newborns may also trigger trained immunity. A key approach to boost vaccine immunogenicity in functionally immunocompromised individuals, such as the very young and the elderly, is the addition of adjuvants. The self-adjuvanted BCG vaccine, live attenuated Mycobacterium bovis, activates multiple TLRs and nucleotide-binding oligomerization domain 2 , triggers trained immunity $(27,39)$, and is associated with reduced all cause mortality, largely due to infections other than tuberculosis, in the first month of life (40).

\section{IMPLICATIONS}

\section{Basic Research/Immunology}

In this review, we have raised the question of whether the current dogma that innate immunity lacks memory characteristics can still be supported. We have provided an overview of a growing body of literature that strongly suggests that innate immune responses have adaptive characteristics. This emerging concept of mechanisms that enable innate immune memory through trained immunity has several crucial implications. First, the discovery of trained immunity broadens the perspective through which we understand host defense to infection. While immune memory has been considered until now as exclusively mediated by specific $\mathrm{T} / \mathrm{B}$-cell responses, the theory of trained immunity posits that that innate immune responses can adapt as well, strengthening, for example, broad NK-, monocyte-, and macrophage-based defense in response to prior infection.

Of note, classical immune memory and trained immunity also differ in fundamental ways. One crucial aspect is that of molecular mechanisms employed: somatic rearrangement vs. epigenetic reprogramming, as detailed above. Another key aspect concerns the specificity of trained immunity. While some studies in invertebrates show specificity in protection against certain pathogens or rejection during transplantation models, most studies in plants and insects reveal heterologous ("nonspecific") effects in which the initial infection protects against a larger array of secondary infections. The protection observed during trained immunity in mammals also suggests that immunological memory of innate immune responses is broad and not limited to a single category of pathogens, and is thus fundamentally different from the classical adaptive immunological memory. It is tempting to speculate that in mammals the specificity of trained immunity was lost in evolution, as its biological relevance diminished, when adaptive immunity conferred the specificity needed during reinfection. The description of adaptive responses within innate immunity will likely lead to important novel insights in the mechanisms of immunomodulation on the one hand, and in defining novel (and more effective) vaccination strategies on the other hand.

\section{Translational Studies}

Trained immunity has important implications for development of novel therapeutic modalities:

"Stand alone" immunomodulators. Molecules that activate the innate immune system via engagement of PRRs can enhance host defense against a range of microbes. Future studies will likely assess the potential of stand-alone PRR agonists, such as TLRAs, to boost host defense against a range of pathogens. For example, pretreatment with TLRAs can enhance defense against polymicrobial sepsis in newborn mice (33). Of note, imiquimod, a TLR7A that activates interferon- $\alpha$ secretion from plasmacytoid dendritic cells (pDCs) is FDA-approved as a topical antiviral against human papilloma virus (warts) and has also been studied off-label for treatment of mulluscipox (41). A rapidly growing number of novel TLRAs have been identified and their activity differs with age (42) such that trained immunity effects may be age specific. The recent recognition of trained immunity may inform future studies of TLRAs to boost host defense in vulnerable populations and 


\section{Implications of innate immune memory $\quad$ Review}

potentially also reduce the risks of allergy and asthma, for example, via balancing Th2/Th1 polarization in accordance with the hygiene hypothesis (43).

Heterologous effects of currently administered vaccines. Vaccines vary in their ability to engage the innate immune system. For example, Alum, the most commonly used vaccine adjuvant, may exert its adjuvant activity via a range of mechanisms, including activation of the inflammasome and release of danger-associated molecular pattern molecules $(44,45)$. Of note, a birth dose of Alum-adjuvanted pneumococcal conjugate vaccine was associated with subsequent Th2-polarization of TLRA-induced mononuclear cell cytokine responses in vitro (46), suggesting that Alum adjuvant may trigger long-term (i.e., at least several months long) Th2-polarized immunity. While Th2 responses may be triggered in a distinct manner from the aforementioned NK-/monocyte-based trained immunity, they do suggest polarization of antigen-presenting cell function through imprinting, likely reflecting epigenetic modulation similar to that contributing to trained immunity. Several studies suggest that BCG may alter responses to other immunizations. For example, as compared to infants who did not receive BCG, BCG-immunized infants have demonstrated higher concentrations of antipneumococcal polysaccharide IgGs (significantly so for serotype $9 \mathrm{~V}$ and $18 \mathrm{C}$ ) and of antiHaemophilus influenzae type b IgG and antitetanus toxoid $\operatorname{IgG}(47)$. In contrast, titers of anti-HBs IgG were lower in the BCG-immunized group than the non-BCG-immunized group.

Development of novel adjuvanted vaccine formulations. Beneficial heterologous effects have been described for live vaccines such as BCG and measles virus vaccine (48). In this context, future vaccine development and clinical trials should take into account not only pathogen-specific immunity, but possibly heterologous immunomodulatory effects, some of which may be mediated by trained immunity. Ideally, novel vaccine formulations may be developed that not only induce pathogen-specific immunity but also have heterologous innate immune-enhancing effects that may reduce the risk of other unrelated infections and/or reduce the risk of allergy and atopy (43).

In conclusion, innate immunity expresses a memory function that underlies broad heterologous immunity to antigenically diverse pathogens. Mechanisms underlying trained immunity include epigenetic reprogramming of monocytes and macrophages, and possible NK cells. Much remains to learned about both the immunologic mechanisms underlying trained immunity and the potential of innate immune memory to elicit broad beneficial effects from select "stand alone" immune response modifiers to novel adjuvanted vaccine formulations.

\section{ACKNOWLEDGMENTS}

O.L. acknowledges the mentorship of Michael Wessels, Richard Malley, and Gary Fleisher.

\section{STATEMENT OF FINANCIAL SUPPORT}

The laboratory of O.L. is funded by the National Institutes of Health (R01Al100135-01) and the Bill \& Melinda Gates Foundation (Global Health Grants OPPGH5284 and OPP1035192). M.G.N. was supported by a Vici grant of the Netherlands Organisation for Scientific Research. O.L.'s laboratory has received sponsored and/or reagent support from VentiRx Pharmaceuticals (San Diego, CA) and 3M Drug Delivery Systems (St. Paul, MN).

\section{REFERENCES}

1. Roitt I, Delves P, Martin SM, Burton DR. Roitt's Essential Immunology, 11th edn. Oxford, UK: Wiley, John \& Sons, Inc, 2006.

2. Akira S, Uematsu S, Takeuchi O. Pathogen recognition and innate immunity. Cell 2006;124:783-801.

3. Fearon DT. Seeking wisdom in innate immunity. Nature 1997;388:323-4.

4. Medzhitov R, Janeway CA Jr. Decoding the patterns of self and nonself by the innate immune system. Science 2002;296:298-300.

5. Kurtz J. Specific memory within innate immune systems. Trends Immunol 2005;26:186-92.

6. Faulhaber LM, Karp RD. A diphasic immune response against bacteria in the American cockroach. Immunology 1992;75:378-81.

7. Arala-Chaves $M$, Sequeira $\mathrm{T}$. Is there any kind of adaptive immunity in invertebrates? Aquaculture 2000;191:247-58.

8. Moret Y, Siva-Jothy MT. Adaptive innate immunity? Responsive-mode prophylaxis in the mealworm beetle, Tenebrio molitor. Proc Biol Sci 2003;270:2475-80.

9. Rheins LA, Karp RD, Butz A. Induction of specific humoral immunity to soluble proteins in the American cockroach (Periplaneta americana). I. Nature of the primary response. Dev Comp Immunol 1980;4: 447-58.

10. Moret Y, Schmid-Hempel P. Immune defence in bumble-bee offspring. Nature 2001;414:506.

11. Witteveldt J, Cifuentes CC, Vlak JM, van Hulten MC. Protection of Penaeus monodon against white spot syndrome virus by oral vaccination. J Virol 2004;78:2057-61.

12. Netea MG, Quintin J, van der Meer JW. Trained immunity: a memory for innate host defense. Cell Host Microbe 2011;9:355-61.

13. Danilova N. The evolution of adaptive immunity. Adv Exp Med Biol 2012;738:218-35.

14. Kurtz J, Armitage SA. Alternative adaptive immunity in invertebrates. Trends Immunol 2006;27:493-6.

15. Dong Y, Taylor HE, Dimopoulos G. AgDscam, a hypervariable immunoglobulin domain-containing receptor of the Anopheles gambiae innate immune system. PLoS Biol 2006;4:e229.

16. Sun JC, Beilke JN, Lanier LL. Adaptive immune features of natural killer cells. Nature 2009;457:557-61.

17. Cooper MA, Elliott JM, Keyel PA, Yang L, Carrero JA, Yokoyama WM. Cytokine-induced memory-like natural killer cells. Proc Natl Acad Sci USA 2009;106:1915-9.

18. Paust S, Senman B, von Andrian UH. Adaptive immune responses mediated by natural killer cells. Immunol Rev 2010;235:286-96.

19. O'Leary JG, Goodarzi M, Drayton DL, von Andrian UH. T cell- and B cell-independent adaptive immunity mediated by natural killer cells. Nat Immunol 2006;7:507-16.

20. Paust S, Gill HS, Wang BZ, et al. Critical role for the chemokine receptor CXCR6 in NK cell-mediated antigen-specific memory of haptens and viruses. Nat Immunol 2010;11:1127-35.

21. Blanden RV, Lefford MJ, Mackaness GB. The host response to CalmetteGuérin bacillus infection in mice. J Exp Med 1969;129:1079-107.

22. Sher NA, Chaparas SD, Greenberg LE, Bernard S. Effects of BCG, Corynebacterium parvum, and methanol-extration residue in the reduction of mortality from Staphylococcus aureus and Candida albicans infections in immunosuppressed mice. Infect Immun 1975;12:1325-30.

23. Tribouley J, Tribouley-Duret J, Appriou M. [Effect of Bacillus Callmette Guerin (BCG) on the receptivity of nude mice to Schistosoma mansoni]. C R Seances Soc Biol Fil 1978;172:902-4.

24. van 't Wout JW, Poell R, van Furth R. The role of BCG/PPD-activated macrophages in resistance against systemic candidiasis in mice. Scand J Immunol 1992;36:713-9. 
25. Garly ML, Martins CL, Balé C, et al. BCG scar and positive tuberculin reaction associated with reduced child mortality in West Africa. A non-specific beneficial effect of BCG? Vaccine 2003;21:2782-90.

26. Rosenthal SR, Loewinsohn E, Graham ML, Liveright D, Thorne MG, Johnson V. BCG vaccination in tuberculous households. Am Rev Respir Dis 1961;84:690-704.

27. Kleinnijenhuis J, Quintin J, Preijers F, et al. Bacille Calmette-Guerin induces NOD2-dependent nonspecific protection from reinfection via epigenetic reprogramming of monocytes. Proc Natl Acad Sci USA 2012;109:17537-42.

28. Bistoni F, Vecchiarelli A, Cenci E, Puccetti P, Marconi P, Cassone A. Evidence for macrophage-mediated protection against lethal Candida albicans infection. Infect Immun 1986;51:668-74.

29. Bistoni F, Verducci G, Perito S, et al. Immunomodulation by a low-virulence, agerminative variant of Candida albicans. Further evidence for macrophage activation as one of the effector mechanisms of nonspecific anti-infectious protection. J Med Vet Mycol 1988;26:285-99.

30. Vecchiarelli A, Cenci E, Puliti M, et al. Protective immunity induced by low-virulence Candida albicans: cytokine production in the development of the anti-infectious state. Cell Immunol 1989;124:334-44.

31. Quintin J, Saeed S, Martens JH, et al. Candida albicans infection affords protection against reinfection via functional reprogramming of monocytes. Cell Host Microbe 2012;12:223-32.

32. Strunk T, Prosser A, Levy O, et al. Responsiveness of human monocytes to the commensal bacterium Staphylococcus epidermidis develops late in gestation. Pediatr Res 2012;72:10-8.

33. Wynn JL, Scumpia PO, Winfield RD, et al. Defective innate immunity predisposes murine neonates to poor sepsis outcome but is reversed by TLR agonists. Blood 2008;112:1750-8.

34. Zhang JP, Yang Y, Levy O, Chen C. Human neonatal peripheral blood leukocytes demonstrate pathogen-specific coordinate expression of TLR2, TLR4/MD2, and MyD88 during bacterial infection in vivo. Pediatr Res 2010;68:479-83.

35. Kronforst KD, Mancuso CJ, Pettengill M, et al. A neonatal model of intravenous Staphylococcus epidermidis infection in mice $<24 \mathrm{~h}$ old enables char acterization of early innate immune responses. PLoS ONE 2012;7:e43897.

36. Kim YM, Romero R, Chaiworapongsa T, et al. Toll-like receptor- 2 and -4 in the chorioamniotic membranes in spontaneous labor at term and in preterm parturition that are associated with chorioamnionitis. Am J Obstet Gynecol 2004;191:1346-55.

37. Strunk T, Doherty D, Jacques A, et al. Histologic chorioamnionitis is associated with reduced risk of late-onset sepsis in preterm infants. Pediatrics 2012;129:e134-41.

38. Wynn JL, Hansen NI, Das A, et al.; Eunice Kennedy Shriver National Institute of Child Health and Human Development Neonatal Research Network. Early sepsis does not increase the risk of late sepsis in very low birth weight neonates. J Pediatr 2013;162:942-8.e1-3.
39. Kleinnijenhuis J, Oosting M, Joosten LA, Netea MG, Van Crevel R. Innate immune recognition of Mycobacterium tuberculosis. Clin Dev Immunol 2011;2011:405310.

40. Aaby P, Roth A, Ravn H, et al. Randomized trial of BCG vaccination at birth to low-birth-weight children: beneficial nonspecific effects in the neonatal period? J Infect Dis 2011;204:245-52.

41. Miller RL, Meng TC, Tomai MA. The antiviral activity of Toll-like receptor 7 and 7/8 agonists. Drug News Perspect 2008;21:69-87.

42. Kollmann TR, Levy O, Montgomery RR, Goriely S. Innate immune function by Toll-like receptors: distinct responses in newborns and the elderly. Immunity 2012;37:771-83.

43. Belderbos M, Levy O, Bont L. Neonatal innate immunity in allergy development. Curr Opin Pediatr 2009;21:762-9.

44. Marrack P, McKee AS, Munks MW. Towards an understanding of the adjuvant action of aluminium. Nat Rev Immunol 2009;9:287-93.

45. Marichal $\mathrm{T}$, Ohata $\mathrm{K}$, Bedoret $\mathrm{D}$, et al. DNA released from dying host cells mediates aluminum adjuvant activity. Nat Med 2011;17:9961002 .

46. van den Biggelaar AH, Richmond PC, Pomat WS, et al. Neonatal pneumococcal conjugate vaccine immunization primes $\mathrm{T}$ cells for preferential Th2 cytokine expression: a randomized controlled trial in Papua New Guinea. Vaccine 2009;27:1340-7.

47. Ritz N, Mui M, Balloch A, Curtis N. Non-specific effect of Bacille CalmetteGuérin vaccine on the immune response to routine immunisations. Vaccine 2013;31:3098-103.

48. Flanagan KL, van Crevel R, Curtis N, Shann F, Levy O; Optimmunize Network. Heterologous ("nonspecific") and sex-differential effects of vaccines: epidemiology, clinical trials, and emerging immunologic mechanisms. Clin Infect Dis 2013;57:283-9.

49. van den Burg HA, Takken FL. Does chromatin remodeling mark systemic acquired resistance? Trends Plant Sci 2009;14:286-94.

50. Conrath U. Molecular aspects of defence priming. Trends Plant Sci 2011;16:524-31

51. Slaughter A, Daniel X, Flors V, Luna E, Hohn B, Mauch-Mani B. Descendants of primed Arabidopsis plants exhibit resistance to biotic stress. Plant Physiol 2012;158:835-43.

52. Durrant WE, Dong X. Systemic acquired resistance. Annu Rev Phytopathol 2004;42:185-209.

53. Jaskiewicz M, Conrath U, Peterhänsel C. Chromatin modification acts as a memory for systemic acquired resistance in the plant stress response. EMBO Rep 2011;12:50-5.

54. Pham LN, Dionne MS, Shirasu-Hiza M, Schneider DS. A specific primed immune response in Drosophila is dependent on phagocytes. PLoS Pathog 2007;3:e26.

55. Rodrigues J, Brayner FA, Alves LC, Dixit R, Barillas-Mury C. Hemocyte differentiation mediates innate immune memory in Anopheles gambiae mosquitoes. Science 2010;329:1353-5. 